\title{
Neural correlates of extrinsic and intrinsic outcome processing during learning in individuals with TBI: a Pilot Investigation
}

Ekaterina Dobryakova ( $\sim$ edobryakova@kesslerfoundation.org)

Kessler Foundation Research Center https://orcid.org/0000-0002-6005-2115

Suzanne Zuckerman

Kessler Foundation

Joshua Sandry

Montclair State University

\section{Research Article}

Keywords: traumatic brain injury, functional MRI, learning, striatum, rehabilitation, feedback

Posted Date: July 1st, 2021

DOI: https://doi.org/10.21203/rs.3.rs-185857/v1

License: (1) This work is licensed under a Creative Commons Attribution 4.0 International License.

Read Full License

Version of Record: A version of this preprint was published at Brain Imaging and Behavior on August 18th, 2021. See the published version at https://doi.org/10.1007/s11682-021-00508-6. 


\section{Abstract}

Objective: Outcome processing, the ability to learn from feedback, is an important component of adaptive behavior and rehabilitation. Evidence from healthy adults implicates the striatum and dopamine in outcome processing. Animal research shows that damage to dopaminergic pathways in the brain can lead to a disruption of dopamine tone and transmission. Such evidence thus suggests that persons with TBI experience deficits in outcome processing. However, no research has directly investigated outcome processing and associated neural mechanisms in TBI. Here, we examine outcome processing in individuals with TBI during learning. Given that TBI negatively impacts striatal and dopaminergic systems, we hypothesize that individuals with TBI exhibit deficits in learning from outcomes.

Methods: To test this hypothesis, individuals with moderate-to-severe TBI and healthy adults were presented with a declarative paired-associate word learning task. Outcomes indicating performance accuracy were presented immediately during task performance and in the form of either monetary or performance-based feedback. Two types of feedback provided the opportunity to test whether extrinsic and intrinsic motivational aspects of outcome presentation play a role during learning and outcome processing.

Results: Our results show that individuals with TBI exhibited impaired learning from feedback compared to healthy participants. Additionally, individuals with TBI exhibited increased activation in the striatum during outcome processing.

Conclusions: The results of this study suggest that outcome processing and learning from immediate outcomes is impaired in individuals with TBI and might be related to inefficient use of neural resources during task performance as reflected by increased activation of the striatum.

\section{Introduction}

Approximately 69 million people sustain a traumatic brain injury (TBI) each year worldwide (Dewan et al., 2019) with cognitive difficulties being extremely common (Jourdan et al., 2018). Memory deficit, resulting from impaired acquisition, is a long-lasting and primary cognitive symptom experienced by individuals with TBI (Carlozzi, Grech, \& Tulsky, 2013; Coste et al., 2015; DeLuca, Schultheis, Madigan, Christodoulou, \& Averill, 2000; Langlois, Rutland-Brown, \& Wald, 2006; Vanderploeg, Donnell, Belanger, \& Curtiss, 2014). Deeper understanding of the impaired learning, memory, and neural mechanisms will lay the prerequisite strong foundation for developing novel rehabilitation treatments for these deficits (Horowitz, Suls, \& Treviño, 2018; Sandry, 2015). Difficulty learning from action outcomes (performance feedback) mediated by the fronto-striatal network is one underexplored and potentially critical area for understanding the underlying causes of memory problems in TBI.

Knowing the outcomes of your actions is a critical component of the learning process. Whether positive or negative, feedback allows learners to modify their behavior in order to achieve a specific goal (Schlund \& Pace, 1999) and acquire new skills. Positive and negative feedback indicate an action's efficacy and 
whether it should be continued or discontinued/replaced, respectively. Feedback-based learning largely relies on the fronto-striatal network, with the ventral striatum and the ventromedial prefrontal cortex (VMPFC) playing critical roles (Tricomi \& Depasque, 2017). Neuroimaging studies have shown that activity in the striatum increases during positive feedback presentation and decreases during negative feedback presentation. Such similar activation patterns are observed during both non-declarative and declarative learning tasks (Tricomi \& Fiez, 2008). Further, this activation pattern has been detected regardless of whether feedback is extrinsic (such as a monetary gain/loss) (e.g. Delgado, Nystrom, Fissell, Noll, \& Fiez, 2000) or intrinsic (such as positive/negative performance related feedback) (Tricomi, Delgado, McCandliss, McClelland, \& Fiez, 2006).

The distinction in feedback type (monetary vs. performance related) is particularly important in the context of rehabilitation. Monetary feedback represents an objective form of feedback where the value of the feedback is clear. This type of feedback has been frequently used in basic cognitive neuroscience investigations (e.g. Delgado et al., 2000; Tricomi, Delgado, \& Fiez, 2004). Performance feedback represents a subjective form of feedback and more closely resembles the type of feedback encountered by individuals in a rehabilitation setting. According to the Cognitive Evaluation Theory (Dec, Ryan, \& Deci, 1999), these two types of feedback differentially impact task performance. Monetary Feedback leads to decreased learning because it undermines one's self-determination and competence, thus reducing intrinsic motivation (i.e. reducing the perception that the activity is inherently rewarding and not dependent on external rewards). Non-monetary performance feedback relies on intrinsic motivation and would not devalue learning, resulting in better performance.

At the neurochemical level, feedback-based learning has been shown to rely on dopamine (BrombergMartin, Matsumoto, \& Hikosaka, 2010; Schultz, 1998), one modulatory neurotransmitter involved in declarative memory formation (Adcock, Thangavel, Whitfield-Gabrieli, Knutson, \& Gabrieli, 2006; Wittmann et al., 2005). Individuals with neurological damage and dopaminergic dysfunction show impaired learning from feedback and altered activation of the striatum and associated regions (du Plessis et al., 2018; Griffiths, Morris, \& Balleine, 2014; van der Vegt et al., 2013). For example, progressive cell death of dopaminergic neurons leads to disrupted learning through feedback in individuals with Parkinson's disease (PD). Specifically, performance accuracy decreases when individuals with PD learn through feedback (Foerde and Shohamy, 2011; Shohamy et al., 2004). Other clinical conditions that are characterized by the fronto-striatal dopaminergic system dysfunction (e.g. schizophrenia and depression), also exhibit deficits in learning from feedback (Griffiths et al., 2014; Pujara \& Koenigs, 2014).

Animal and human models suggest a similar neurochemical profile may be evident after TBI. Rats given controlled cortical impact injury, a model of closed-head TBI, show altered dopamine neurotransmission (Wagner et al., 2005) and progressive loss of nigrostriatal neurons (Hutson et al., 2011). Relatedly, dopamine transmission after controlled cortical impact improves with the administration of dopamine agonists such as methylphenidate (Wagner, Drewencki, et al., 2009; Wagner, Sokoloski, et al., 2009) and bromocriptine (Kline, Massucci, Marion, \& Dixon, 2002). Administration of these types of medications in individuals with TBI also leads to improvements in cognitive 
functioning (Bales, Kline, Wagner, \& Dixon, 2010; Bales, Wagner, Kline, \& Dixon, 2009; Garcia, Shah, Dixon, Wagner, \& Kline, 2011). In-line with these findings, behavioral evidence suggests that individuals with TBI demonstrate impaired outcome anticipation (e.g. Freedman, Bleiberg, \& Freedland, 1987) and impaired learning of action-outcome contingencies (Larson, Kelly, Stigge-Kaufman, Schmalfuss, \& Perlstein, 2007; Schlund, 2002; Schlund, Pace, \& McGready, 2001), both of which rely on the dopaminergic system.

Additional support for striatal dysfunction in TBI comes from structural magnetic resonance imaging (MRI). Persons with TBI show increased diffusivity in the striatum related to impulsive decision-making (i.e. deciding what action to perform to get a desired outcome) (Newcombe et al., 2011) and compromised striatal white matter tracts are linked to executive dysfunction (Shah et al., 2012). Striatal volume is also reduced in individuals with severe TBI and striatal abnormalities have been reported in mild TBI (Rangaprakash et al., 2017; Tate et al., 2016). This evidence suggests that individuals with TBI may experience negative cognitive symptoms resulting from striatal dysregulation.

Despite strong parallels between PD and TBI that implicate striatum-mediated learning dysfunction (Jenkins et al., 2020) and consistent previous evidence of learning impairments in TBI (DeLuca et al., 2000; Vanderploeg et al., 2014; Wright, Schmitter-Edgecombe, \& Woo, 2010), no studies have directly examined the functioning of the striatum in individuals with TBI and whether individuals with TBI exhibit deficits in learning through feedback. To address this gap, we investigated 1) whether individuals with TBI are able to learn from feedback and 2) the role the striatum plays in feedback learning. We hypothesized that individuals with TBI will exhibit worse learning through feedback compared to HCs. In addition, we contrasted two alternative neural hypotheses to account for impairments in learning from feedback in TBI. Specifically, compared to HCs, individuals with TBI could exhibit decreased striatal activation during feedback processing. This would be consistent with PD literature and findings of the fronto-striatal network being sensitive to task success (DePasque Swanson \& Tricomi, 2014; Dobryakova \& Tricomi, 2013; Tricomi et al., 2006). Alternatively, compared to HCs, individuals with TBI could exhibit increased striatal activation during feedback processing. This would be consistent with previous studies that observed increased brain activation in neurological populations and suggest that such hyperactivation results from inefficient use of neural resources during task performance (Manning, 2008; Poston et al., 2016; Turner, Mclntosh, \& Levine, 2011).

To test the above hypothesis, participants engaged in a paired-associate learning paradigm (Dobryakova \& Tricomi, 2013; Tricomi \& Fiez, 2008), where they first studied word associations outside of the MRI. Next, during functional MRI (fMRI) data acquisition, participants' memory for the paired associates was tested, with either monetary or performance feedback provided for some of the stimuli. This direct feedback manipulation allowed us to investigate the degree that different feedback types (internal vs. external) may modulate striatal activation and learning in TBI.

\section{Methods}


Participants. Seventeen participants with moderate-to-severe TBI and 25 healthy control (HC) participants consented to participate in this pilot study. The Wechsler Abbreviated Scale of Intelligence (WASI) Vocabulary subtest was used to characterize premorbid verbal ability (not collected on 5 participants with $\mathrm{TBI}$ and $1 \mathrm{HC}$; see Table 1 for demographic information). Participants were recruited through advertisements/word of mouth and the TBI Model System (TBIMS) compiled into a database of individuals interested in TBI research. According to the TBIMS, TBI is defined as "damage to brain tissue caused by an external mechanical force, as evidenced by loss of consciousness due to brain trauma, post-traumatic amnesia, skull fracture, or objective neurological findings that can reasonably be attributed to TBI on physical examination or mental status examination". Moderate-to-severe injury severity is defined as posttraumatic amnesia lasting more than $24 \mathrm{hrs}$, loss of consciousness lasting more than 30 min, Glasgow Coma Scale (GCS) score less than 13. Participants had no history of psychiatric or neurological diseases aside from $\mathrm{TBI}$ and no reported history of substance abuse or dependence. TBI participants were at least 1-year post injury (chronic stage of TBI) and able to undergo MRI. TBI severity was determined at participants' acute care hospital as per medical records based on the duration of loss of consciousness, duration of post-traumatic amnesia, Glasgow Coma Scale, and/or positive neuroimaging findings. Diagnosis was done either by a medical care provider in an acute hospital setting $(n=7)$, medical care provider with specialized knowledge of TBI $(n=9)$, and/or a clinical neuropsychologist $(n=1)$. The research was approved by the Institutional Review Board of Kessler Foundation.

\section{Procedure}

Scan session. Participants received an fMRI scan of the brain conducted on a Siemens Skyra 3T scanner (see Appendix for details).

Behavioral paradigm. The learning task was adapted from Dobryakova and Tricomi (2013) and consist of the Study Phase, the Feedback Phase, and the Test Phase (Figure 1A).

Study Phase. Outside of the scanner participants learned word associations (180 trials). On each trial, participants were presented with three words: a target word with two alternative options underneath (See Appendix for details). One alternative was highlighted in green indicating that this option is the correct match to the target word. Participants were instructed to memorize the target word and highlighted paired-associate. Each trial was presented for $4 \mathrm{sec}$ in random order.

Feedback Phase. Study Phase words were randomly assigned to one of three feedback conditions presented during the fMRI scan: Monetary Feedback, Non-Monetary Feedback, and No Feedback. A cue screen was presented with the target word positioned above two choice words. Participants selected the choice word that matched the target word based on what they remembered from the Study Phase. In the Monetary Feedback condition, a correct match resulted in feedback of a green circle with the monetary gain amount written inside of it (\$1.00); an incorrect match resulted in feedback of a red circle with a monetary loss amount written inside of it (\$0.50). Participants were informed that they will receive a bonus based on their performance in this condition. The Non-Monetary Feedback condition resembled 
the Monetary Feedback condition with the exception of no monetary gains or losses. In the No Feedback condition, participants were presented with a blue circle after making their choice. Conditions were randomly intermixed in blocks of 10 trials, starting with a jittered fixation point (1-5 s) that contained a label informing participants of the current condition. This was followed by a cue (4sec) and feedback screen (1 sec) (Figure 1B). Trial order was also randomized.

Test phase. The final Test Phase occurred outside of the scanner. Studied paired-associates were represented to participants for $4 \mathrm{sec}$ each in random order. Participants were asked to select the correct answer.

After finishing, all participants were compensated $\$ 150$ regardless of their performance.

Post-task Questionnaire. After the MRI scan, participants completed a post-experimental questionnaire to evaluate subjective group differences in task-engagement, feedback related affect, and preference for positive and negative feedback. Specifically, we asked: 1) whether participants were engaged while performing the task, and 2) how did they feel when being presented with positive and negative feedback.

\section{Statistical analysis}

Behavioral Data. Accuracy served as the main dependent measure (See Appendix for details). Accuracy data from the Feedback Phase were analyzed with mixed effects ANOVA with Group (HC, TBI) as a between subject factor and Phase (Feedback, Test) and Feedback condition (Monetary Feedback, NonMonetary Feedback, No Feedback) as within subject factors. Additional analyses included a 2 Phase X 3 Feedback condition repeated measures ANOVA, computed independently for each group. We present Cohen's d estimate of effect size alongside independent and paired t-test simple comparisons. Analyses were computed using R v3.3.2.

fMRI data. Standard fMRI data preprocessing and analysis were carried out using FEAT (FMRI Expert Analysis Tool) v6.00 of FSL (See Appendix for preprocessing details).

The second level analysis, which averaged contrast estimates over runs per subject, was carried out using a fixed effects model by forcing the random effects variance to zero in FLAME (FMRIB's Local Analysis of Mixed Effects) (Siegel et al., 2014; Smith, 2002; Woolrich, Ripley, Brady, \& Smith, 2001). Group analysis was carried out using FLAME stage 1. To correct for multiple comparisons, Z statistical images were thresholded using a cluster threshold of $z>2.3$ and a corrected cluster significance threshold of p<0.05 (Worsley, 2001).

To address the main research question and examine the role of the striatum during feedback learning in individuals with TBI, we first examined whether there are differences between groups during feedback presentation and performed a contrast that collapsed across feedback type (i.e. regressors from the Monetary Feedback and Non-Monetary Feedback conditions were combined): TBI positive feedback > HC positive feedback. We then performed contrasts on identified voxels to compare parameter estimates associated with positive Monetary Feedback and positive Non-Monetary Feedback conditions between 
groups: 1) TBI positive Monetary Feedback > HC positive Monetary Feedback, 2) TBI positive NonMonetary Feedback > HC positive Non-Monetary Feedback. Negative feedback was analyzed in the same manner.

\section{Results}

Behavioral Accuracy. TBI participants performed worse and did not learn from feedback compared to the $\mathrm{HC}$ group as indicated by the Feedback condition by Group interaction that approached significance, $F(2,80)=2.99, p=.056$ (Figure 2). The Feedback condition main effect, $F(2,80)=4.00, p=.022$, and the Feedback condition $X$ Phase interaction were also significant, $F(2,80)=4.50, p=.014$. No other main effects or interactions were significant. Follow-up simple comparisons of the interaction, collapsing across Phase, also approached significance and exhibited a large effect sizes, revealing that the HCs performed better than TBI participants when presented with Monetary Feedback $[t(40)=1.99, p=.054$, $d=.62]$ and when presented with Non-Monetary Feedback $[t(40)=1.95, p=.058, d=.61]$. The groups did not differ on the No Feedback condition $[t(40)=.83, p=.41, d=.26]$.

We further evaluated performance for each group independently using a 2 Phase $X 3$ Feedback condition repeated measures ANOVA to better understand nuances of the data pattern as a function of Group. For the HCs, the interaction between Phase and Feedback condition was significant, $F(2,48)=3.70, p=.03$ (Figure 3-top). Specifically, during the Test Phase, performance in the Monetary Feedback condition $[t(24)=5.98, p<.001, d=.36]$ and the Non-Monetary Feedback condition $[t(24)=5.04, p<.001, d=.40]$ was better than performance in the No Feedback condition. Monetary Feedback and Non-Monetary Feedback conditions did not differ, $\mathrm{t}(24)=.62, \mathrm{p}=.54, \mathrm{~d}=.05$. The interaction was driven by a difference between the Feedback and Test Phase for the Non-Monetary condition $[t(24)=2.94, p=.007, d=.34]$. There were no differences between the Feedback and Test Phases for the Monetary Feedback condition $[t(24)=1.60$, $p=.12, d=.17]$ and for the No Feedback condition $[t(24)=.43, p=.12, d=.05]$. The Phase X Feedback interaction was not significant for the TBI Group, $F(2,32)=1.34, p=.28$, verifying that TBI participants did not learn from feedback, while HCs did.

fMRI results. To determine the regions sensitive to feedback processing during learning in individuals with $\mathrm{TBI}$ vs. HC, we first performed a contrast to identify voxels sensitive to positive feedback presentation. That is, while collapsing across feedback conditions, positive feedback presentation was contrasted between TBI and HC groups: TBI (positive Monetary Feedback + positive Non-Monetary Feedback) > HC (positive Monetary Feedback + positive Non-Monetary Feedback). This comparison revealed extensive activation in the TBI vs. HCs in the striatum and the VMPFC as well as other cortical regions (Table 2; Figure 4A). A comparison of HCs vs. TBI did not reveal any significant activation clusters. Identical comparison of negative feedback presentation (TBI [negative monetary feedback + negative non-monetary feedback] > HC [negative monetary feedback + negative non-monetary feedback]) did not reveal any activation differences suggesting the effect was restricted to positive feedback. 
Since we had a strong a priori hypothesis focused specifically on the striatum (Dobryakova \& Tricomi, 2013; Tricomi \& Fiez, 2008), we performed a between group comparison for Monetary and Non-Monetary Feedback conditions on voxels of the right and left anterior caudate (cluster extent of 448 and 40 voxels, respectively) showing sensitivity to positive feedback presentation. Significant differences between HCs and TBI were detected bilaterally. TBIs showed greater activation in the right anterior caudate $(\mathrm{t}(40)=3.07$, $p<0.005)$, and left anterior caudate, $(t(40)=2.48, p<0.05)$ when processing feedback (Figure $4 b, 4 c)$.

Post-task Questionnaire. See Appendix for details.

\section{Discussion}

Learning impairment is one of the most commonly observed disabilities in individuals with $\mathrm{TBI}$ and is identified as a major component in the breakdown of the acquisition process that facilitates memory (DeLuca et al., 2000; Vakil, 2005). One prerequisite for many rehabilitation interventions is for patients to be able to learn a rehabilitation strategy after receiving feedback from a therapist. Implicit in this assumption is that individuals with $\mathrm{TBI}$ are able to process the outcomes of their actions (i.e. feedback) so that they can learn from them. This is a requirement for cognitive rehabilitation as well as motor rehabilitation due to individuals relying on feedback or outcomes before an action becomes automatic.

Inability to learn from feedback can thus exert a significant negative impact on both physical and cognitive rehabilitation after TBI. The inability to learn from feedback in TBI may contribute to perseveration of incorrect actions and/or inefficacy of compensatory strategy use. Perseveration, or the inability to stop repetition of a spceific behavior is a common consequence of TBI and is part of the inhibition and impulse control symptomology in TBI (Selzer, Clarke, Cohen, Duncan, \& Gage, 2006). In the current task, it can manifest as selecting an incorrect resonponse even after the receipt of negative feedback. Successful acquisition of compensatory strategies during rehabilitation also requires abandoning a strategy that is not working and swithching to a strategy that results in positive outcomes. The current findings also have implications in scenarious where a therapist is involved as the findings potentially suggest that indivudlas with TBI are not able to fully utilize the feedback provided by a therapist during rehablitation in order to learn a compensatory strategy.

In the current study, we directly examined whether individuals with TBI exhibit learning deficits due to impaired outcome processing by presenting participants with TBI and HCs with a paired-associate word leaning task and different types of feedback intended to improve their learning. Our data show that participants with moderate-to-severe TBI exhibit deficits in learning through feedback compared to HCs. Consistent with past research, learning in the HCs group improved with non-monetary (subjective) feedback with a similar but non-significant pattern of performance for monetary (objective) feedback (Albrecht, Abeler, Weber, \& Falk, 2014; Daniel \& Pollmann, 2010; Gawlowska et al., 2017). This was not the case in participants with TBI whose learning did not improve, regardless of whether monetary or non-monetary feedback was presented. These results suggest that, while individuals with TBI might be able to acquire information as well as HCs in this paired-associate learning paradigm (as we did not 
observe group differences in accuracy during the Feedback Phase), feedback does not improve their learning and memory beyond what participants originally learned (i.e. no improvements in accuracy at Test Phase in the TBI group). These data parallel the findings from individuals with PD who also show deficits in learning from immediate feedback compared to HCs (Foerde, Race, Verfaellie, \& Shohamy, 2013).

Our analysis of neuroimaging data revealed greater activation in the TBI group vs. the HC group in several cortical brain regions as well as in the striatum. Such 'overactivity' can be interpreted as disinhibition or as a reflection of increased in cognitive demands (Price \& Friston, 1999) that individuals with TBI experience during task performance. Such findings are consistent with previous investigations that interpret increased brain activity in other neurological populations (Manning, 2008; Poston et al., 2016; Turner et al., 2011) as well as in TBI (Hillary et al., 2014) as inefficient use of neural resources during task performance.

The functioning of the striatum was of particular relevance in the current study. Contrary to previous findings in action-outcome literature of increased striatal activation in association with improvements in learning in conjunction with increased striatal activation (Dobryakova \& Tricomi, 2013; Hiebert et al., 2014; O'Doherty, 2004), we observed increased striatal activation and poor learning in the TBI group compared to HCs. However, greater striatal activation to feedback presentation in the TBI group might reflect the rewarding nature of feedback rather than the learning signal in the striatum. Receiving feedback has been shown to be similarly rewarding as receiving reinforcers such as money (DePasque \& Tricomi, 2015; Tricomi et al., 2006). Indeed, most TBI participants indicated that they felt very happy when presented with positive feedback (see Appendix). Thus, increased activation of the striatum in the absence of learning might suggest that, while the striatum does not carry the learning signal, feedback presentation is still rewarding to individuals with TBI. Increased activation of the striatum may also indicate its role in maintaining the representation of the rewards' value throughout the duration of the task (Beck, Locke, Savine, Jimura, \& Braver, 2010).

The insula and the anterior cingulate cortex (ACC) that also showed increased activation in the TBI vs. HC groups in the current study have been implicated in a variety of goal-directed tasks as well as in tasks that require mental effort (Engström, Karlsson, Landtblom, \& Craig, 2014) and reward processing (Heilbronner \& Hayden, 2016). Activation of these regions in the current study might suggest that, compared to HC participants, participants with TBI exerted more cognitive effort (Spirou, Chiaravalloti, \& Dobryakova, 2018) to process immediate positive feedback and to hold on to the information depicted by positive feedback. This interpretation aligns well with other studies showing ACC involvement in information retention and its role in storing information that is required for appropriate action performance (Heilbronner \& Hayden, 2016). The insula is frequently coactivated with the ACC and the medial prefrontal cortex (that also showed increased activation in the present study) during studies involving goal-directed behavior and has reciprocal anatomical connections with the ACC as well as the striatum (Gogolla, 2017; Haber, 2011; Haber, Fudge, \& McFarland, 2000). The insula is often affected by 
neurological and psychiatric disorders and is implicated in a variety of symptoms as well as dopaminedependent learning and memory (Gogolla, 2017).

Given the importance of outcome processing during learning and rehabilitation for individuals with TBI, future studies should examine whether feedback processing can be improved though normalizing striatal activation. Previous studies in non-TBI individuals suggest that striatal activation is malleable to "normalization" (Dahlin, Neely, Larsson, Bäckman, \& Nyberg, 2008; Gavelin et al., 2017). Similarly, animal literature also suggests that pharmacological interventions can normalize striatal functioning through administration of dopamine agonist (Krishna, Beitchman, Bromberg, \& Thomas, 2020; Wagner, Drewencki, et al., 2009).

Limitations. The current study provides important new evidence about learning through feedback and mechanisms involved in outcome processing in TBI. However, it has several limitations. While the effect sizes were large in this study, future replication in a larger sample is necessary to verify the statistical patterns that were observed. One additional limitation is that WASI Vocabulary scores were unavailable for a small subset of participants. It is possible that premorbid verbal ability differed between groups and this was not captured as a result of missing data. Further, the Monetary Feedback condition in the current task reflects not only performance feedback but also carries an objective value. While previous investigations used a similar design (Daniel \& Pollmann, 2010) and we did not observed differences in brain activity between Monetary and Non-Monetary Feedback conditions, future studies should utilize an experimental task that separates the value and the learning signal to further explain striatal activation in the absence of improved learning in TBI.

\section{Conclusions}

The current pilot study examined learning through feedback in individuals with TBI and associated brain activity. Results revealed impairments during learning through feedback in individuals with TBI that are similar to previously documented findings in PD (Foerde et al., 2013). In conjunction with poor learning from feedback, we also observed increased brain activation that might suggest inefficient use of neural resources during task performance in individuals with TBI. Such impairment may contribute to observable memory disability and poor rehabilitation outcomes in individuals with TBI. Moreover, neural and cognitive mechanisms of impaired feedback learning may serve as a modifiable treatment target.

\section{Declarations}

Funding sources: This work was supported by the New Jersey Health Foundation (\#PC103-17) and the National Multiple Sclerosis Society (RG-1501-02630), awarded to Ekaterina Dobryakova, PhD.

Conflict of Interest: The authors report no conflict of interest.

Ethics approval: The research was approved by the Institutional Review Board of Kessler Foundation. 
Consent to participate: All participants consented to participate in this research.

Consent for publication: All authors consent for publication of this material.

Availability of data and material: Available upon request.

Code availability: Not applicable

Author Contributions: ED: conceptualization, fMRI data analysis, original manuscript draft preparation; SZ: editing, behavioral data analysis, table and figure preparation; JS: behavioral data analysis, figure preparation, manuscript preparation and editing.

\section{References}

Adcock, R. A., Thangavel, A., Whitfield-Gabrieli, S., Knutson, B., \& Gabrieli, J. D. E. (2006). RewardMotivated Learning: Mesolimbic Activation Precedes Memory Formation. Neuron, 50(3), 507-517. https://doi.org/10.1016/j.neuron.2006.03.036

Albrecht, K., Abeler, J., Weber, B., \& Falk, A. (2014). The brain correlates of the effects of monetary and verbal rewards on intrinsic motivation. Frontiers in Neuroscience, 8, 303.

https://doi.org/10.3389/fnins.2014.00303

Bales, J. W., Kline, A. E., Wagner, A. K., \& Dixon, C. E. (2010). Targeting Dopamine in Acute Traumatic Brain Injury. The Open Drug Discovery Journal, 2, 119-128. https://doi.org/10.2174/1877381801002010119

Bales, J. W., Wagner, A. K., Kline, A. E., \& Dixon, C. E. (2009). Persistent cognitive dysfunction after traumatic brain injury: A dopamine hypothesis. Neuroscience and Biobehavioral Reviews, 33(7), 9811003. https://doi.org/10.1016/j.neubiorev.2009.03.011

Beck, S. M., Locke, H. S., Savine, A. C., Jimura, K., \& Braver, T. S. (2010). Primary and secondary rewards differentially modulate neural activity dynamics during working memory. PloS One, 5(2), e9251. https://doi.org/10.1371/journal.pone.0009251

Bromberg-Martin, E. S., Matsumoto, M., \& Hikosaka, O. (2010). Dopamine in motivational control:

rewarding, aversive, and alerting. Neuron, 68(5), 815-834. https://doi.org/10.1016/j.neuron.2010.11.022

Carlozzi, N. E., Grech, J., \& Tulsky, D. S. (2013). Memory functioning in individuals with traumatic brain injury: An examination of the Wechsler Memory Scale-Fourth Edition (WMS-IV). Journal of Clinical and Experimental Neuropsychology. https://doi.org/10.1080/13803395.2013.833178

Coltheart, M. (1981). The MRC psycholinguistic database. Quarterly Journal of Experimental Psychology, A 33, 497-505. 
Coste, C., Navarro, B., Vallat-Azouvi, C., Brami, M., Azouvi, P., \& Piolino, P. (2015). Disruption of temporally extended self-memory system following traumatic brain injury. Neuropsychologia. https://doi.org/10.1016/j.neuropsychologia.2015.03.014

Dahlin, E., Neely, A. S., Larsson, A., Bäckman, L., \& Nyberg, L. (2008). Transfer of learning after updating training mediated by the striatum. Science (New York, N.Y.), 320(5882), 1510-1512. https://doi.org/10.1126/science.1155466

Daniel, R., \& Pollmann, S. (2010). Comparing the neural basis of monetary reward and cognitive feedback during information-integration category learning. The Journal of Neuroscience: The Official Journal of the Society for Neuroscience, 30(1), 47-55. https://doi.org/10.1523/JNEUROSCI.2205-09.2010

Dec, E. L., Ryan, R. M., \& Deci, E. L. (1999). A Meta-Analytic Review of Experiments Examining the Effects of Extrinsic Rewards on Intrinsic Motivation. In Psychological Bulletin (Vol. 125). Retrieved from http://citeseerx.ist.psu.edu/viewdoc/download?doi=10.1.1.588.5821\&rep=rep1\&type=pdf

Delgado, M., Nystrom, L. E., Fissell, C., Noll, D. C., \& Fiez, J. A. (2000). Tracking the hemodynamic responses to reward and punishment in the striatum. Journal of Neuroph, 84, 3072-3077.

DeLuca, J., Schultheis, M. T., Madigan, N. K., Christodoulou, C., \& Averill, A. (2000). Acquisition versus retrieval deficits in traumatic brain injury: implications for memory rehabilitation. Archives of Physical Medicine and Rehabilitation, 81(10), 1327-1333. https://doi.org/10.1053/apmr.2000.9390

DePasque, S., \& Tricomi, E. (2015). Effects of intrinsic motivation on feedback processing during learning. Neurolmage, 119, 175-186. https://doi.org/10.1016/j.neuroimage.2015.06.046

DePasque Swanson, S., \& Tricomi, E. (2014). Goals and task difficulty expectations modulate striatal responses to feedback. Cognitive, Affective \& Behavioral Neuroscience, 14(2), 610-620. https://doi.org/10.3758/s13415-014-0269-8

Dewan, M. C., Rattani, A., Gupta, S., Baticulon, R. E., Hung, Y. C., Punchak, M., ... Park, K. B. (2019). Estimating the global incidence of traumatic brain injury. Journal of Neurosurgery, 130(4), 1080-1097. https://doi.org/10.3171/2017.10.JNS17352

Dobryakova, E., \& Tricomi, E. (2013). Basal ganglia engagement during feedback processing after a substantial delay. Cognitive, Affective \& Behavioral Neuroscience, 13(4), 725-736.

https://doi.org/10.3758/s13415-013-0182-6

du Plessis, S., Bossert, M., Vink, M., van den Heuvel, L., Bardien, S., Emsley, R., ... Carr, J. (2018). Reward processing dysfunction in ventral striatum and orbitofrontal cortex in Parkinson's disease. Parkinsonism and Related Disorders, 48, 82-88. https://doi.org/10.1016/j.parkreldis.2017.12.024

Engström, M., Karlsson, T., Landtblom, A.-M., \& Craig, A. D. B. (2014). Evidence of conjoint activation of the anterior insular and cingulate cortices during effortful tasks. Frontiers in Human Neuroscience, 8 , 
Foerde, K., Race, E., Verfaellie, M., \& Shohamy, D. (2013). A role for the medial temporal lobe in feedbackdriven learning: evidence from amnesia. The Journal of Neuroscience: The Official Journal of the Society for Neuroscience, 33(13), 5698-5704. https://doi.org/10.1523/JNEUROSCI.5217-12.2013

Freedman, P. E., Bleiberg, J., \& Freedland, K. (1987). Anticipatory behaviour deficits in closed head injury. Journal of Neurology Neurosurgery and Psychiatry, 50(4), 398-401.

https://doi.org/10.1136/jnnp.50.4.398

Garcia, A. N., Shah, M. A., Dixon, C. E., Wagner, A. K., \& Kline, A. E. (2011). Biologic and plastic effects of experimental traumatic brain injury treatment paradigms and their relevance to clinical rehabilitation. $P M$ \& R: The Journal of Injury, Function, and Rehabilitation, 3(6 Suppl 1), S18-27.

https://doi.org/10.1016/j.pmrj.2011.03.017

Gavelin, H. M., Neely, A. S., Andersson, M., Eskilsson, T., Järvholm, L. S., \& Boraxbekk, C. J. (2017). Neural activation in stress-related exhaustion: Cross-sectional observations and interventional effects.

Psychiatry Research - Neuroimaging, 269(August), 17-25.

https://doi.org/10.1016/j.pscychresns.2017.08.008

Gawlowska, M., Beldzik, E., Domagalik, A., Gagol, A., Marek, T., \& Mojsa-Kaja, J. (2017). I Don't Want to Miss a Thing - Learning Dynamics and Effects of Feedback Type and Monetary Incentive in a Paired Associate Deterministic Learning Task. Frontiers in Psychology, 8, 935.

https://doi.org/10.3389/fpsyg.2017.00935

Gogolla, N. (2017, June 19). The insular cortex. Current Biology, Vol. 27, pp. R580-R586. https://doi.org/10.1016/j.cub.2017.05.010

Greve, D. N., \& Fischl, B. (2009). Accurate and robust brain image alignment using boundary-based registration. Neurolmage, 48(1), 63-72. https://doi.org/10.1016/j.neuroimage.2009.06.060

Griffiths, K. R., Morris, R. W., \& Balleine, B. W. (2014). Translational studies of goal-directed action as a framework for classifying deficits across psychiatric disorders. Frontiers in Systems Neuroscience, 8(May), 1-16. https://doi.org/10.3389/fnsys.2014.00101

Haber, S. (2011). Neuroanatomy of Reward: A View from the Ventral Striatum. In Neurobiology of Sensation and Reward. Retrieved from http://www.ncbi.nlm.nih.gov/pubmed/22593898

Haber, S., Fudge, J. L., \& McFarland, N. R. (2000). Striatonigrostriatal pathways in primates form an ascending spiral from the shell to the dorsolateral striatum. Journal of Neuroscience, 20(6), 2369-2382.

Heilbronner, S. R., \& Hayden, B. Y. (2016). Dorsal Anterior Cingulate Cortex: A Bottom-Up View. Annual Review of Neuroscience, 39, 149-170. https://doi.org/10.1146/annurev-neuro-070815-013952 
Hiebert, N. M., Vo, A., Hampshire, A., Owen, A. M., Seergobin, K. N., \& MacDonald, P. A. (2014). Striatum in stimulus-response learning via feedback and in decision making. Neurolmage, 101, 448-457. https://doi.org/10.1016/j.neuroimage.2014.07.013

Hillary, F. G., Rajtmajer, S. M., Roman, C. a., Medaglia, J. D., Slocomb-Dluzen, J. E., Calhoun, V. D., ... Wylie, G. R. (2014). The rich get richer: Brain injury elicits hyperconnectivity in core subnetworks. PLoS ONE, 9(8). https://doi.org/10.1371/journal.pone.0104021

Horowitz, T. S., Suls, J., \& Treviño, M. (2018, August 1). A Call for a Neuroscience Approach to CancerRelated Cognitive Impairment. Trends in Neurosciences, Vol. 41, pp. 493-496. https://doi.org/10.1016/j.tins.2018.05.001

Hutson, C. B., Lazo, C. R., Mortazavi, F., Giza, C. C., Hovda, D., \& Chesselet, M.-F. (2011). Traumatic brain injury in adult rats causes progressive nigrostriatal dopaminergic cell loss and enhanced vulnerability to the pesticide paraquat. Journal of Neurotrauma, 28(9), 1783-1801.

https://doi.org/10.1089/neu.2010.1723

Jenkins, P. O., Roussakis, A. A., De Simoni, S., Bourke, N., Fleminger, J., Cole, J., ... Sharp, D. (2020). Distinct dopaminergic abnormalities in traumatic brain injury and Parkinson's disease. Journal of Neurology, Neurosurgery and Psychiatry, 91(6), 631-637. https://doi.org/10.1136/jnnp-2019-321759

Jenkinson, M., Bannister, P., Brady, M., \& Smith, S. (2002). Improved Optimization for the Robust and Accurate Linear Registration and Motion Correction of Brain Images. Neurolmage, 17(2), 825-841. https://doi.org/10.1006/nimg.2002.1132

Jourdan, C., Azouvi, P., Genêt, F., Selly, N., Josseran, L., \& Schnitzler, A. (2018). Disability and Health Consequences of Traumatic Brain Injury. American Journal of Physical Medicine \& Rehabilitation, 97(5), 323-331. https://doi.org/10.1097/PHM.0000000000000848

Kline, A. E., Massucci, J. L., Marion, D. W., \& Dixon, C. E. (2002). Attenuation of working memory and spatial acquisition deficits after a delayed and chronic bromocriptine treatment regimen in rats subjected to traumatic brain injury by controlled cortical impact. Journal of Neurotrauma, 19(4), 415-425. https://doi.org/10.1089/08977150252932370

Krishna, G., Beitchman, J. A., Bromberg, C. E., \& Thomas, T. C. (2020, January 2). Approaches to monitor circuit disruption after traumatic brain injury: Frontiers in preclinical research. International Journal of Molecular Sciences, Vol. 21. https://doi.org/10.3390/ijms21020588

Landauer, T. K., Foltz, P. W., \& Laham, D. (1998). An introduction to latent semantic analysis. Discourse Processes, 25(2-3), 259-284.

Langlois, J. A., Rutland-Brown, W., \& Wald, M. M. (2006). The epidemiology and impact of traumatic brain injury: a brief overview. The Journal of Head Trauma Rehabilitation, 21(5), 375-378. 
https://doi.org/00001199-200609000-00001 [pii]

Larson, M. J., Kelly, K. G., Stigge-Kaufman, D. A., Schmalfuss, I. M., \& Perlstein, W. M. (2007). Reward context sensitivity impairment following severe TBI: an event-related potential investigation. Journal of the International Neuropsychological Society: JINS, 13(4), 615-625.

https://doi.org/10.1017/S1355617707070762

Manning, L. (2008). Do some neurological conditions induce brain plasticity processes? Behavioural Brain Research, 192(1), 143-148. https://doi.org/10.1016/j.bbr.2008.04.001

Newcombe, V. F. J., Outtrim, J. G., Chatfield, D. A., Manktelow, A., Hutchinson, P. J., Coles, J. P., ... Menon, D. K. (2011). Parcellating the neuroanatomical basis of impaired decision-making in traumatic brain injury. Brain: A Journal of Neurology, 134(Pt 3), 759-768. https://doi.org/10.1093/brain/awq388

O'Doherty, J. P. (2004). Reward representations and reward-related learning in the human brain: insights from neuroimaging. Current Opinion in Neurobiology, 14(6), 769-776.

https://doi.org/10.1016/j.conb.2004.10.016

Poston, K. L., YorkWilliams, S., Zhang, K., Cai, W., Everling, D., Tayim, F. M., ... Menon, V. (2016). Compensatory neural mechanisms in cognitively unimpaired Parkinson disease. Annals of Neurology, 79(3), 448-463. https://doi.org/10.1002/ana.24585

Price, C. J., \& Friston, K. J. (1999). Scanning Patients With Tasks They Can Perform. Human Brain Mapping, 8, 102-108.

Pujara, M., \& Koenigs, M. (2014). Mechanisms of reward circuit dysfunction in psychiatric illness: prefrontal-striatal interactions. The Neuroscientist: A Review Journal Bringing Neurobiology, Neurology and Psychiatry, 20(1), 82-95. https://doi.org/10.1177/1073858413499407

Rangaprakash, D., Deshpande, G., Daniel, T. A., Goodman, A. M., Robinson, J. L., Salibi, N., ... Dretsch, M. N. (2017). Compromised hippocampus-striatum pathway as a potential imaging biomarker of mildtraumatic brain injury and posttraumatic stress disorder. Human Brain Mapping, 38(6), 2843-2864. https://doi.org/10.1002/hbm.23551

Sandry, J. (2015). Working memory and memory loss in neurodegenerative disease. Neurodegenerative Disease Management, 5(1), 1-4. https://doi.org/10.2217/nmt.14.51

Schlund, M. W. (2002). The effects of brain injury on choice and sensitivity to remote consequences: deficits in discriminating response-consequence relations. Brain Injury, 16(4), 347-357. https://doi.org/10.1080/02699050110103977

Schlund, M. W., \& Pace, G. (1999). Relations between traumatic brain injury and the environment: feedback reduces maladaptive behaviour exhibited by three persons with traumatic brain injury. Brain Injury, 13(11), 889-897. Retrieved from http://www.ncbi.nlm.nih.gov/pubmed/10579660 
Schlund, M. W., Pace, G. M., \& McGready, J. (2001). Relations between decision-making deficits and discriminating contingencies following brain injury. Brain Injury, 15(12), 1061-1071.

https://doi.org/10.1080/02699050110086887

Schultz, W. (1998). The phasic reward signal of primate dopamine neurons. Advances in Pharmacology (San Diego, Calif.), 42, 686-690. Retrieved from http://www.ncbi.nlm.nih.gov/pubmed/9327992

Selzer, M., Clarke, S., Cohen, L., Duncan, P., \& Gage, F. (2006). Textbook of Neural Repair and Rehabilitation. In M. Selzer, S. Clarke, L. Cohen, P. Duncan, \& F. Gage (Eds.), Textbook of Neural Repair and Rehabilitation. https://doi.org/10.1017/cbo9780511995590

Shah, S., Yallampalli, R., Merkley, T. L., Mccauley, S. R., Bigler, E. D., Macleod, M., ... Wilde, E. A. (2012). Diffusion tensor imaging and volumetric analysis of the ventral striatum in adults with traumatic brain injury. Brain Injury, 26(3), 201-210. https://doi.org/10.3109/02699052.2012.654591

Siegel, J. S., Power, J. D., Dubis, J. W., Vogel, A. C., Church, J. A., Schlaggar, B. L., \& Petersen, S. E. (2014). Statistical improvements in functional magnetic resonance imaging analyses produced by censoring high-motion data points. Human Brain Mapping, 35(5), 1981-1996. https://doi.org/10.1002/hbm.22307

Smith, S. M. (2002). Fast robust automated brain extraction. Human Brain Mapping, 17(3), 143-155. https://doi.org/10.1002/hbm.10062

Spirou, A., Chiaravalloti, N. D., \& Dobryakova, E. (2018). Corticostriatal Hyperactivation to Reward Presentation in Individuals With TBI With High Depressive Symptomatology: A Pilot Study. Journal of Head Trauma and Rehabilitation. https://doi.org/10.1097/HTR.0000000000000482

Tate, D. F., Wade, B. S. C., Velez, C. S., Drennon, A. M., Bolzenius, J., Gutman, B. A., ... York, G. E. (2016). Volumetric and shape analyses of subcortical structures in United States service members with mild traumatic brain injury. Journal of Neurology, 33(2), 113-122. https://doi.org/10.1007/s00415-016-8236-7

Tricomi, E., Delgado, M. R., \& Fiez, J. A. (2004). Modulation of caudate activity by action contingency. Neuron, 41(2), 281-292. https://doi.org/10.1016/S0896-6273(03)00848-1

Tricomi, E., Delgado, M. R., McCandliss, B. D., McClelland, J. L., \& Fiez, J. A. (2006). Performance feedback drives caudate activation in a phonological learning task. Journal of Cognitive Neuroscience, 18(6), 1029-1043.

Tricomi, E., \& Depasque, S. (2017). The Role Of Feedback In Learning And Motivation. In S. Kim, J. Reeve, \& M. Bong (Eds.), Recent Developments in Neuroscience Research on Human Motivation (Advances in Motivation and Achievement (Vol. 19, pp. 175-202). https://doi.org/10.1108/S0749-

742320160000019015

Tricomi, E., \& Fiez, J. a. (2008). Feedback signals in the caudate reflect goal achievement on a declarative memory task. Neurolmage, 41(3), 1154-1167. https://doi.org/10.1016/j.neuroimage.2008.02.066 
Turner, G. R., Mclntosh, A. R., \& Levine, B. (2011). Prefrontal Compensatory Engagement in TBI is due to Altered Functional Engagement Of Existing Networks and not Functional Reorganization. Frontiers in Systems Neuroscience, 5, 9. https://doi.org/10.3389/fnsys.2011.00009

Vakil, E. (2005). The effect of moderate to severe traumatic brain injury (TBI) on different aspects of memory: a selective review. Journal of Clinical and Experimental Neuropsychology, 27(8), 977-1021. https://doi.org/10.1080/13803390490919245

van der Vegt, J. P. M., Hulme, O. J., Zittel, S., Madsen, K. H., Weiss, M. M., Buhmann, C., ... Siebner, H. R. (2013). Attenuated neural response to gamble outcomes in drug-naive patients with Parkinson's disease. Brain, 136(4), 1192-1203. https://doi.org/10.1093/brain/awt027

Vanderploeg, R. D., Donnell, A. J., Belanger, H. G., \& Curtiss, G. (2014). Consolidation deficits in traumatic brain injury: the core and residual verbal memory defect. Journal of Clinical and Experimental Neuropsychology, 36(1), 58-73. https://doi.org/10.1080/13803395.2013.864600

Wagner, A. K., Drewencki, L. L., Chen, X., Santos, F. R., Khan, A. S., Harun, R., ... Dixon, C. E. (2009). Chronic methylphenidate treatment enhances striatal dopamine neurotransmission after experimental traumatic brain injury. Journal of Neurochemistry, 108(4), 986-997. https://doi.org/10.1111/j.14714159.2008.05840.x

Wagner, A. K., Sokoloski, J. E., Chen, X., Harun, R., Clossin, D. P., Khan, A. S., ... Dixon, C. E. (2009). Controlled cortical impact injury influences methylphenidate-induced changes in striatal dopamine neurotransmission. Journal of Neurochemistry, 110(3), 801-810. https://doi.org/10.1111/j.14714159.2009.06155.x

Wagner, A. K., Sokoloski, J. E., Ren, D., Chen, X., Khan, A. S., Zafonte, R. D., ... Dixon, C. E. (2005). Controlled cortical impact injury affects dopaminergic transmission in the rat striatum. Journal of Neurochemistry, 95(2), 457-465. https://doi.org/10.1111/j.1471-4159.2005.03382.x

Wittmann, B. C., Schott, B. H., Guderian, S., Frey, J. U., Heinze, H. J., \& Duzel, E. (2005). Reward-related fMRI activation of dopaminergic midbrain is associated with enhanced hippocampus-dependent longterm memory formation. Neuron, 45(3), 459-467.

Woolrich, M. W., Ripley, B. D., Brady, M., \& Smith, S. M. (2001). Temporal Autocorrelation in Univariate Linear Modeling of FMRI Data. Neurolmage, 14(6), 1370-1386. https://doi.org/10.1006/nimg.2001.0931

Worsley, K. J. (2001). Statistical Analysis of Activation Images. In P. Jezzard, M. PM, \& S. Smith (Eds.), Functional MRI: An Introduction to Methods (pp. 251-270). New York, NY: Oxford University Press.

Wright, M. J., Schmitter-Edgecombe, M., \& Woo, E. (2010). Verbal memory impairment in severe closed head injury: The role of encoding and consolidation. Journal of Clinical and Experimental Neuropsychology, 32(7), 728-736. https://doi.org/10.1080/13803390903512652 


\section{Tables}

Table 1. Data are presented as means (standard deviation). To allow group comparison on neurocognive function, premorbid verbal IQ was assessed using the Wechsler Abbreviated Scale of Intelligence (WASI) Vocabulary subtest (scores were not collected for 5 TBI and 1 HC participants).

\begin{tabular}{llll} 
& TBI & HC & Statistic (df) \\
\hline Age & $44.29(11.18)$ & $43.36(6.51)$ & $\mathrm{t}(23.4)=.32, \mathrm{p}=.76$ \\
Years of Education & $14.65(1.97)$ & $15.64(2.26)$ & $\mathrm{t}(40)=1.48, \mathrm{p}=.15$ \\
\hline Percent Female & $65 \%$ & $28 \%$ & $\mathrm{X}^{2}(1)=5.56, \mathrm{p}=.02^{*}$ \\
\hline Months Since Injury & $94.41(64.72)$ & & \\
WASI & $50.75(10.68)$ & $50.13(11.37)$ & $\mathrm{t}(34)=.16, \mathrm{p}=.88$ \\
\hline
\end{tabular}

*Sex did not significantly interact with dependent variables and is thus not included in the analyses.

Table 2. Brain regions showing greater sensitivity to feedback presentation in the TBI vs. HC group: [TBI (positive Monetary Feedback + positive Non-Monetary Feedback) > HC (positive Monetary Feedback + positive Non-Monetary Feedback)] $(p<0.05$, corrected).

\begin{tabular}{|c|c|c|c|c|c|c|}
\hline ggion & $\begin{array}{c}\text { \# of } \\
\text { Voxels }\end{array}$ & Hemisphere & $\begin{array}{l}\text { Peak } \\
\text { Z }\end{array}$ & $\begin{array}{l}\text { Peak } \\
\text { X }\end{array}$ & $\begin{array}{l}\text { Peak } \\
\text { Y }\end{array}$ & $\begin{array}{l}\text { Peak } \\
\text { Z }\end{array}$ \\
\hline iterior Cingulate Cortex & 5604 & $\mathrm{R}$ & 5.39 & 4 & 42 & 24 \\
\hline $\begin{array}{l}\text { Ierior Front } \\
\text { rrus/Insula }\end{array}$ & 2555 & $\mathrm{~L}$ & 4.92 & -28 & 24 & -8 \\
\hline iddle Temporal Gyrus & 1088 & $\mathrm{R}$ & 5.48 & 66 & -26 & -8 \\
\hline $\begin{array}{l}\text { ferior Frontal } \\
\text { rrus/Insula }\end{array}$ & 1082 & $\mathrm{R}$ & 4.61 & 30 & 30 & -4 \\
\hline isiform Gyrus (BA 37) & 807 & $\mathrm{~L}$ & 4.64 & -52 & -40 & -14 \\
\hline itate nucleus/Cerebellum & 785 & $\mathrm{~L}$ & 3.92 & -16 & -60 & -20 \\
\hline sterior Cingulate Gyrus & 409 & $\mathrm{R}$ & 4.29 & 6 & -20 & 38 \\
\hline
\end{tabular}

\section{Appendix}

\section{Procedure}

Scan session. 34 functional slices were collected using an EPI sequence (TR $=2,000 \mathrm{~ms}, \mathrm{TE}=30 \mathrm{~ms}$, FOV $=204 \mathrm{~mm}$, flip angle $=90^{\circ}, 3 \times 3 \times 3 \mathrm{~mm}$ voxels) tilted $30^{\circ}$ from the AC-PC line to reduce signal drop-out in the VMPFC (Deichmann, Gottfried, Hutton, \& Turner, 2003). A T1-weighted pulse sequence was used to collect structural images $\left(\mathrm{TR}=2,100 \mathrm{~ms}, \mathrm{TE}=3.43 \mathrm{~ms}\right.$, flip angle $=9^{\circ}, 1 \times 1 \times 1 \mathrm{~mm}$ voxels $)$.

Behavioral paradigm. The words used in the experiment contained 4-8 letters and 1-2 syllables, had Kucera-Francis frequencies of 20-650 words per million, and had high imagibility ratings (score of over 400 according to the MRC database) (Coltheart, 1981). The words were matched for word length and 
frequency at the trial level. Words presented on the same trial were not semantically related, with a score of less than 0.2 on the Latent Semantic Analysis similarity matrix (Landauer, Foltz, \& Laham, 1998), did not rhyme and did not begin with the same letter.

\section{Statistical analysis}

Behavioral data. Outliers were operationalized as trials with response times shorter than $300 \mathrm{~ms}$ as this short response likely indicated an anticipation error. This procedure resulted in removal of $2.43 \%$ (Feedback Phase) and $4.74 \%$ (Test Phase) of trials. A programming error resulted in a few stimulus items in the Feedback Phase being redrawn, leading to some participants $(\mathrm{N}=16)$ viewing the same word twice. These trials were discarded from all analyses (6.03 percent of all trials) and the error was corrected for the remaining participants.

fMRI data: preprocessing. Registration of the functional data to the high resolution structural data was performed using boundary-based registration method (Greve \& Fischl, 2009). High resolution structural to MNI 2mm standard space registration was performed using FLIRT and further refined with FNIRT nonlinear registration (Greve \& Fischl, 2009; Jenkinson, Bannister, Brady, \& Smith, 2002). The following pre-statistics processing were applied: motion correction using MCFLIRT (Jenkinson et al., 2002), slicetiming correction using Fourier-space time-series phase-shifting, non-brain removal using BET (Smith, 2002), spatial smoothing using a Gaussian kernel of FWHM $6.0 \mathrm{~mm}$, grand-mean intensity normalization of the entire 4D dataset by a single multiplicative factor, and high-pass temporal filtering (Gaussianweighted least-squares straight line fitting, with sigma=45.0s). Time-series statistical analysis was carried out using FILM with local autocorrelation correction (Woolrich et al., 2001). The time series model included regressors corresponding to the $1 \mathrm{sec}$ time period of feedback presentation convolved with the double-gamma hemodynamic response function, and their temporal derivatives. A separate regressor was used for each high motion TR, determined using fsl_motion_outliers according to FD > 0.09 (Siegel et al., 2014). Extended motion parameters were included as regressors of no interest (18 regressors: 6 motion parameters, derivatives of the original motion parameters and the squares of the derivatives).

\section{Results}

Post-task Questionnaire. The post-task questionnaire that participants completed after the MRI session served as manipulation check. On the post-task questionnaire, 10 out of 17 participants with TBI indicated that they were engaged while performing the task. More than half indicated feeling happy when presented with positive feedback (12 out of 17) and unhappy when presented with negative feedback (11 out of 17). 20 out of $25 \mathrm{HC}$ participants indicated that they were engaged while performing the task. Most indicated feeling happy when presented with positive feedback (22 out of 25) and unhappy when 
presented with negative feedback (16 out of 25 ). These results demonstrate that the manipulation was effective: positive feedback was perceived as more rewarding and negative feedback was perceived as more punishing/aversive to participants.

\section{Figures}

A.

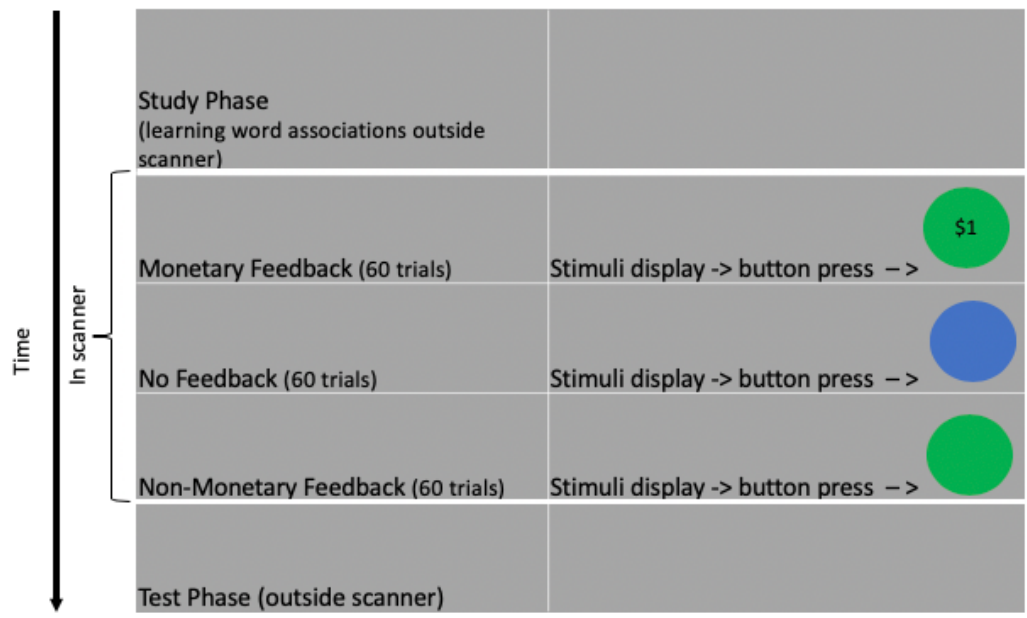

B.

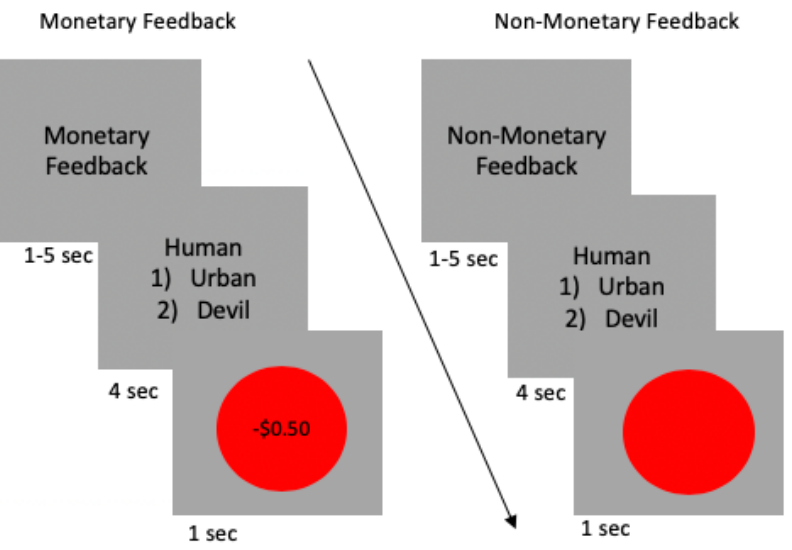

Figure 1. A. Chart of experimental events demonstrating their progression through time. B. Depiction of loss/incorrect trials for the Monetary and NonMonetary Feedback conditions during scanning.

\section{Figure 1}

A. Chart of experimental events demonstrating their progression through time. B. Depiction of loss/incorrect trials for the Monetary and Non-Monetary Feedback conditions during scanning. 


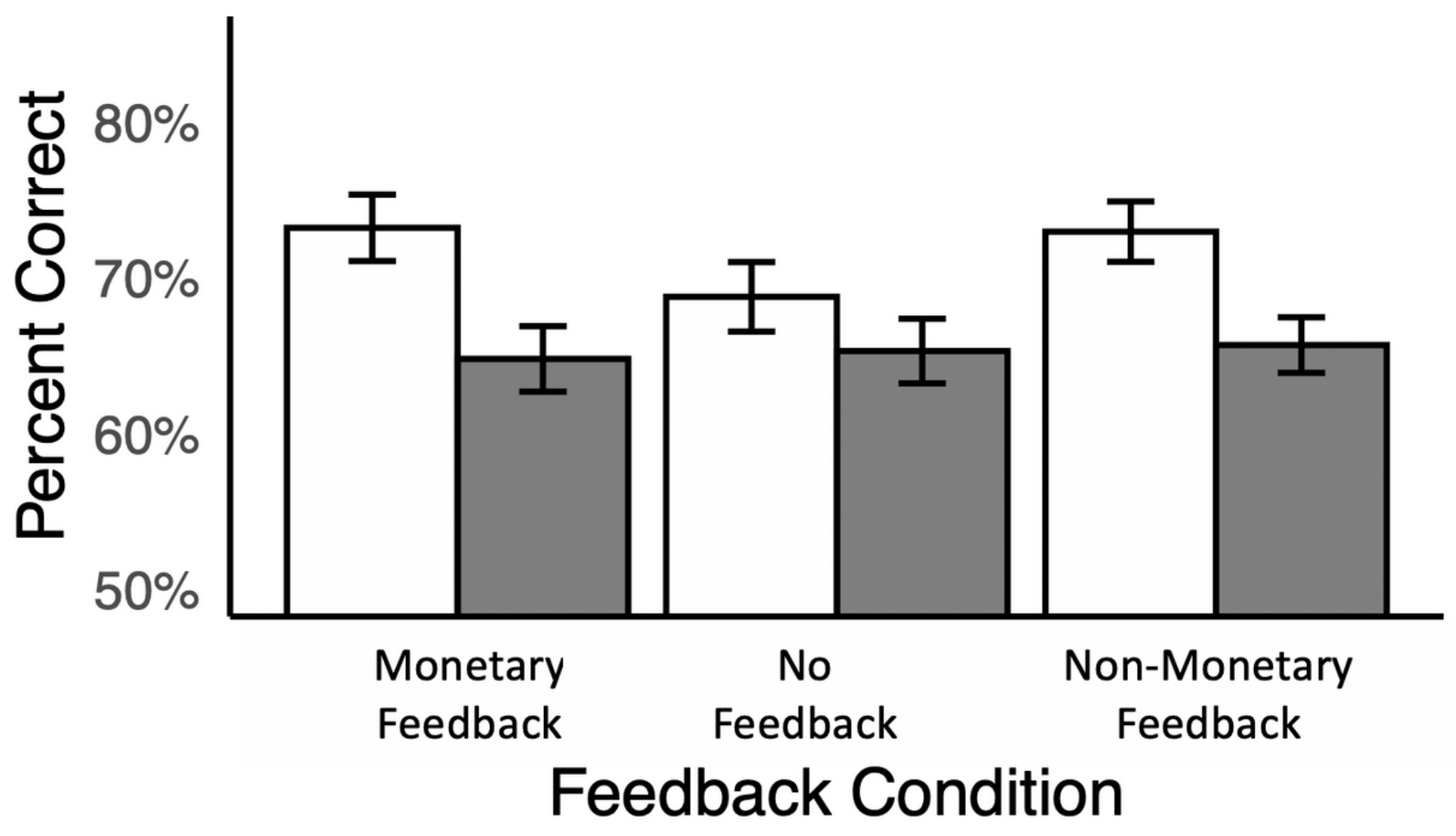

\section{$\square$ Healthy Control $\square$ Traumatic Brain Injury}

Figure 2

Accuracy for the three feedback conditions as a function of group (collapsing across Phase). 


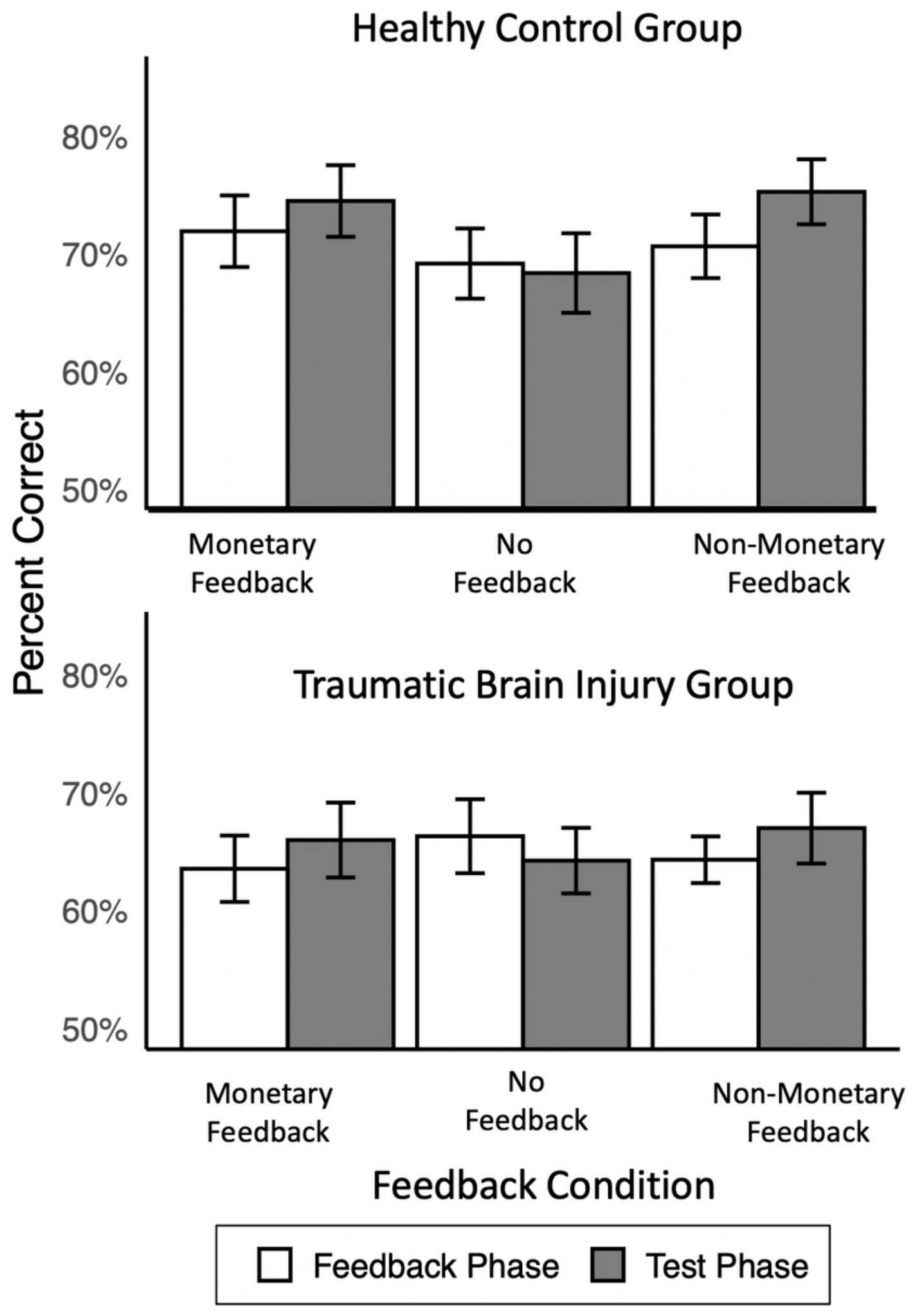

Figure 3

Accuracy for the three Feedback conditions and Phase as a function of HC (top panel) and TBI group (bottom panel). 
A.

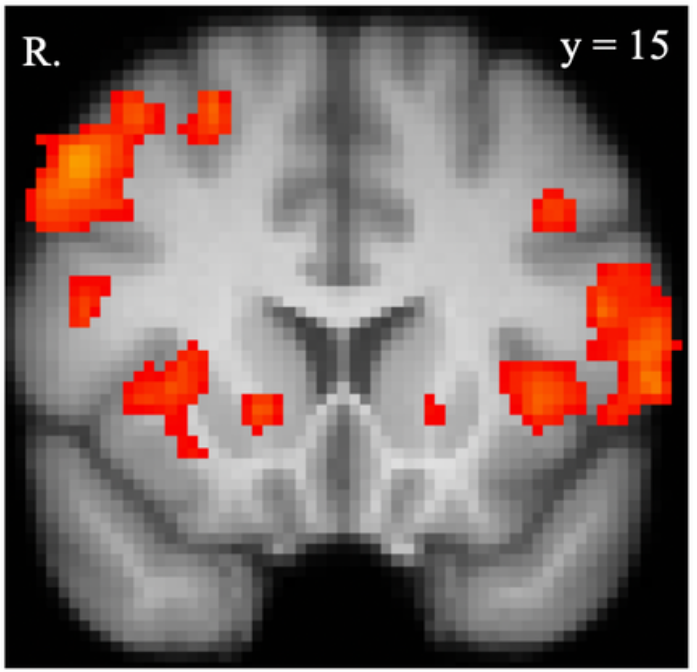

B.

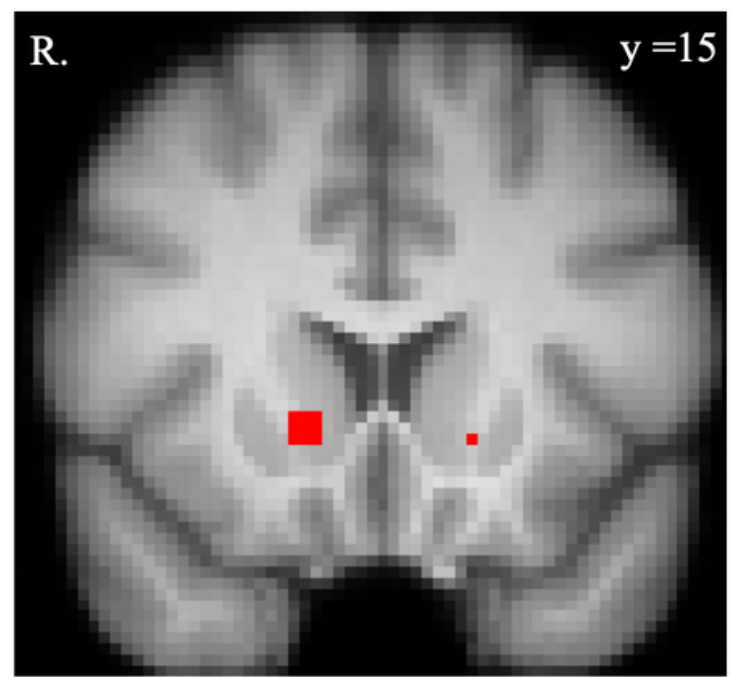

C.

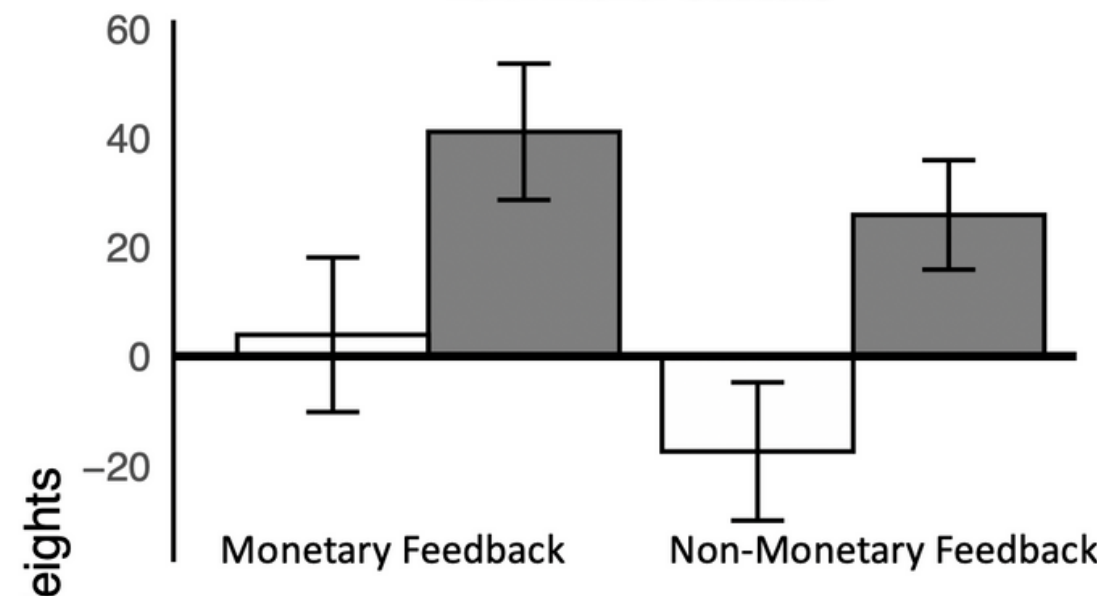

Right Anterior Caudate

胥

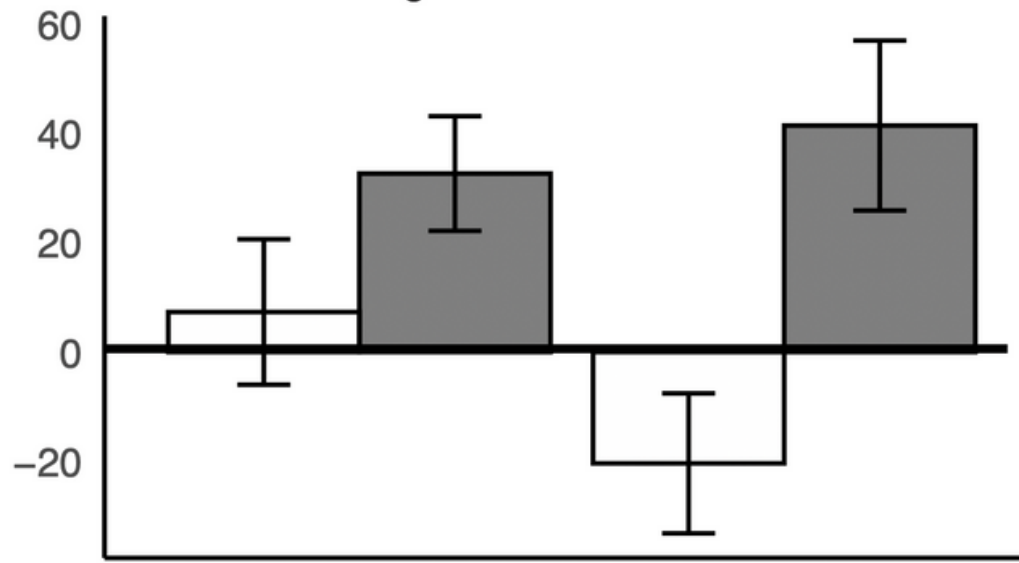

Monetary Feedback

Non-Monetary Feedback

Feedback Condition

Traumatic Brain Injury

Figure 4

A. Brain activity showing sensitivity to positive feedback in TBI vs. HC group (collapsed across the feedback type) ( $p<0.05$, corrected for multiple comparisons). B. Right and left anterior caudate clusters used to extract beta weights for TBI vs. HC comparison of Monetary and Non-Monetary Feedback. C. For illustrative purposes, beta weights from the voxels of the right and left anterior caudate showing an effect of $\mathrm{TBI}>\mathrm{HC}$ during Non-Monetary Feedback presentation. 